\title{
Prognostic relevance of an epigenetic biomarker panel in sentinel lymph nodes from colon cancer patients
}

Guro E. Lind ${ }^{1,2,3^{*}}$ (D) Marianne Guriby ${ }^{1,2,4}$, Terje Ahlquist ${ }^{1,2}$, Israr Hussain ${ }^{5}$, Marine Jeanmougin ${ }^{1,2,3}$, Kjetil Søreide ${ }^{6,7,8}$, Hartwig Kørner ${ }^{6,7}$, Ragnhild A. Lothe ${ }^{1,2,3 \dagger}$ and Oddmund Nordgård ${ }^{5^{*}+}$

\begin{abstract}
Background: Patients with early colorectal cancer (stages I-II) generally have a good prognosis, but a subgroup of 15-20\% experiences relapse and eventually die of disease. Occult metastases have been suggested as a marker for increased risk of recurrence in patients with node-negative disease. Using a previously identified, highly accurate epigenetic biomarker panel for early detection of colorectal tumors, we aimed at evaluating the prognostic value of occult metastases in sentinel lymph nodes of colon cancer patients.

Results: The biomarker panel was analyzed by quantitative methylation-specific PCR in primary tumors and 783 sentinel lymph nodes from 201 patients. The panel status in sentinel lymph nodes showed a strong association with lymph node stage $(P=8.2 \mathrm{E}-17)$. Compared with routine lymph node diagnostics, the biomarker panel had a sensitivity of 79\% (31/39). Interestingly, among 162 patients with negative lymph nodes from routine diagnostics, $13(8 \%)$ were positive for the biomarker panel. Colon cancer patients with high sentinel lymph node methylation had an inferior prognosis (5-year overall survival $P=3.0 \mathrm{E}-4$; time to recurrence $P=3.1 \mathrm{E}-4$ ), although not significant. The same trend was observed in multivariate analyses $(P=1.4 \mathrm{E}-1$ and $P=6.7 \mathrm{E}-2$, respectively). Occult sentinel lymph node metastases were not detected in early stage (I-II) colon cancer patients who experienced relapse.

Conclusions: Colon cancer patients with high sentinel lymph node methylation of the analyzed epigenetic biomarker panel had an inferior prognosis, although not significant in multivariate analyses. Occult metastases in TNM stage II patients that experienced relapse were not detected.
\end{abstract}

Keywords: Biomarkers, DNA methylation, Occult metastases, Prognosis, Relapse, Sentinel lymph nodes

\section{Background}

Colorectal cancer is a frequent disease, ranking third among the most common cancers in men and second in women. The mortality is high, with an estimated number of cancer deaths approaching 700,000 per year, worldwide [1]. Histopathological tumor staging by the tumor-node-metastasis (TNM) system remains the best prognostic marker for colorectal cancer, with lymph

\footnotetext{
*Correspondence: guro.elisabeth.lind@rr-research.no; nood@sus.no

${ }^{\dagger}$ Equal contributors

'Department of Molecular Oncology, Institute for Cancer Research, The Norwegian Radium Hospital, Oslo University Hospital, PO Box 4950 Nydalen, 0424 Oslo, Norway

${ }^{5}$ Department of Hematology and Oncology, Stavanger University Hospital, PO Box 8100, 4068 Stavanger, Norway

Full list of author information is available at the end of the article
}

node status as the strongest prognostic factor. Currently, lymph node status is used for clinical decision-making regarding adjuvant chemotherapy [2]. Despite this, stage alone has clear limitations in terms of treatment stratification, resulting in both under- and over-treatment of patients [3, 4].

Colon cancer patients with TNM stage III are routinely offered postoperative fluoropyrimidine-based chemotherapy, which decreases the risk of death by 10-15\% compared to surgery alone [2]. In contrast, no statistically significant survival benefit has been found for adjuvant chemotherapy in patients with stage II disease. Except for patients with accepted clinical high risk features, such as a low number of lymph nodes examined, tumor perforation, T4 lesions, 
or lack of clean resection margins, stage II patients are not routinely offered adjuvant chemotherapy [5]. However, approximately $15-20 \%$ of patients with stage II disease will experience relapse [6]. Thus, occult metastases in lymph node-negative patients have been suggested as a potential marker for the systemic spread of tumor cells $[4,7,8]$. Consequently, correct identification of the stage II patients that are at risk of recurrence and potentially would benefit from adjuvant chemotherapy would be highly valuable in a clinical perspective.

Epigenetic aberrations, including promoter DNA hypermethylation, is frequently seen during colorectal cancer development $[9,10]$, and several such biomarkers have been suggested for the early detection of this disease. High accuracy is important and best achieved by a panel of individually well-performing biomarkers compared to any single marker alone [11]. Previously, we have identified a highly sensitive and specific panel of DNA methylation biomarkers for colorectal tumors [12-14]. The aim of the present study was twofold: (A) to evaluate the prognostic value of this biomarker panel in ex vivo-sampled sentinel lymph nodes from colon cancer patients and (B) to investigate whether the markers can identify early stage (I-II) patients at high risk of relapse, by identifying occult metastases in histologically negative sentinel lymph nodes.

\section{Results}

DNA methylation biomarker panel status of colon cancers Among the 197 colon cancer patients who had primary tumor biopsies available for analysis, 193 (98\%) were positive for the DNA methylation biomarker panel. The cancer biomarker panel status was associated with neither microsatellite instability (MSI) status nor BRAF mutation status, although all cancers negative for the biomarker panel $(n=4)$ were MSS and had no BRAF V600E hotspot mutation.

\section{DNA methylation biomarker panel status of lymph nodes Normal lymph nodes}

The 43 normal lymph nodes collected from patients with benign bowel disease had generally low levels of DNA promoter methylation (one could not be determined). The mean percent methylated reference (PMR) value across the biomarker panel in the test series of normal lymph nodes was 0.23 , range 0 to 0.64. The threshold for scoring samples as biomarker panel positive was set to the closest upper integer across the test series, PMR $\geq 1$. The validation series had a mean PMR value in the same range as the test series (mean 0.10 , range 0 to 0.45 ). All normal lymph node controls were negative for the DNA methylation biomarker panel (Fig. 1).

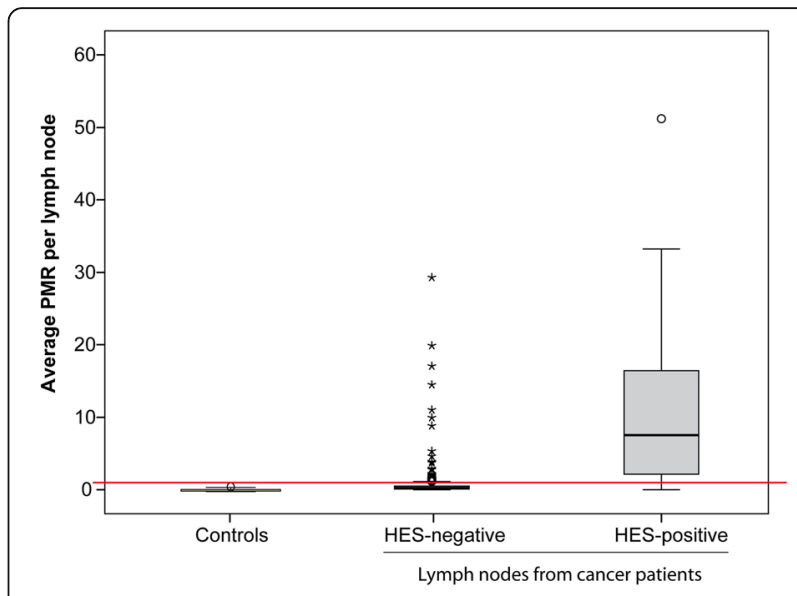

Fig. 1 Average DNA methylation across the biomarker panel for individual lymph nodes. Controls: mean 0.18; 95\% Cl [0.13-0.23]. HES negative: mean $0.52 ; 95 \% \mathrm{Cl}$ [0.40-0.64]. HES positive: mean 10.73; 95\% [Cl 7.84-13.62]. The red line indicates the scoring threshold (1.0). Abbreviations: Cl confidence interval, HES hematoxylin-erythrosinsafranin, PMR percentage methylated reference (methylation value)

\section{Lymph nodes from cancer patients}

Among the 782 successfully analyzed sentinel lymph nodes from colon cancer patients (one sentinel lymph node could not be determined), 92 (12\%) were positive for the DNA methylation biomarker panel. There was no statistically significant difference in the methylation frequency between the test and validation series. In subsequent statistical analyses, the two series were pooled for robustness. Across the series, there was a strong association with hematoxylin-erythrosin-safranin (HES) staining: $43 / 54(80 \%)$ of the HES-positive lymph nodes were positive for the DNA methylation biomarkers $(P=1.3 \mathrm{E}-34$; Fisher's exact). In addition, $49(7 \%)$ of the 726 HES-negative samples were methylation-positive $(P=1.3 \mathrm{E}-34$; Additional file 1: Figure S1a). The average PMR values across the DNA methylation biomarker panel for the individual lymph nodes are visualized in Fig. 1. Based on receiver operating characteristics (ROC) curve analysis, the average PMR values per lymph node, across the DNA methylation biomarker panel, could separate HES-positive from HES-negative patient lymph nodes with an area under the ROC curve (AUC) of 0.914 (asymptotic significance 2.9E-24; Fig. 2).

DNA methylation biomarker panel status of lymph nodes at the patient level

Based on the test series of normal lymph node controls, patients with a methylation index $\geq 1$ were considered lymph node methylation-positive, whereas patients with a methylation index $<1$ were considered to have an unmethylated lymph node status. Among 201 patients with colon cancer, $44(22 \%)$ were positive for the DNA methylation biomarker panel in the analyzed lymph 


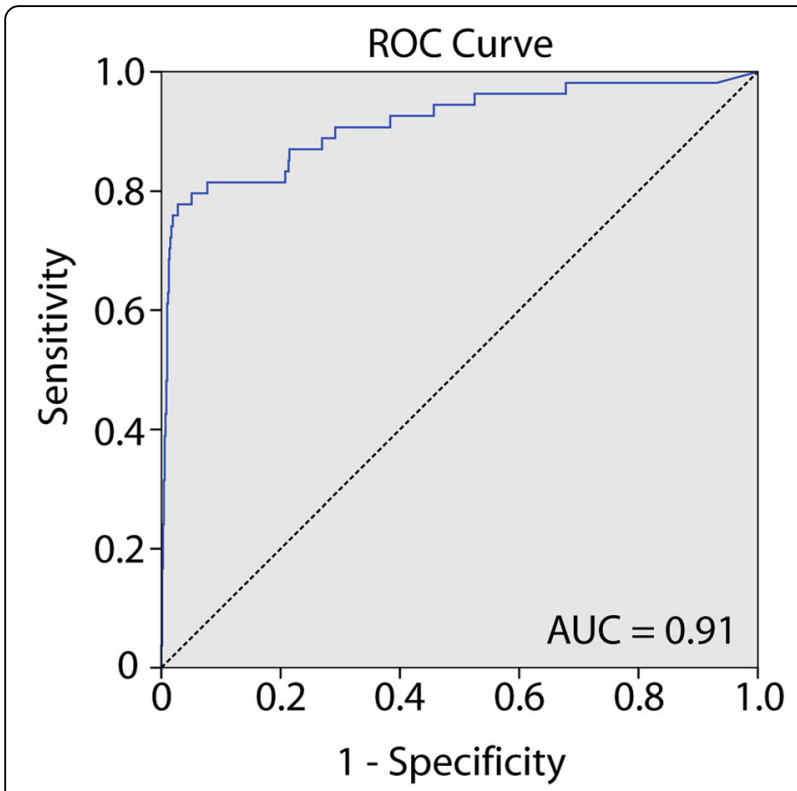

Fig. 2 Receiver operating characteristics curve of the ability of the biomarker panel to separate HES-positive from HES-negative lymph nodes from colon cancer patients. Abbreviations: AUC area under the receiver operating characteristics curve, HES hematoxylin-erythrosin-safranin

nodes. The distribution of HES-positive patients and methylation biomarkers was similar to the distribution seen for individual lymph nodes: $31 / 39$ (79\%) of the patients with one or more HES-positive lymph node(s) analyzed were also biomarker panel positive. Interestingly, $13(8 \%)$ of the 162 patients with only HES-negative lymph nodes were methylation-positive (Additional file 1: Figure S1B). Furthermore, patients in which one or more of the analyzed lymph nodes were HES-positive had significantly higher lymph node methylation index (mean 5.24, 95\% CI [3.80-6.67]) than did patients with HESnegative lymph nodes (mean 0.45, 95\% CI [0.34-0.57], $P<1$ E-4, Mann-Whitney $U$; Fig. 3). Finally, based on ROC curve analysis, the methylation index could distinguish colon cancer patients with positive HES from patients with negative HES with a resulting AUC value of 0.895 (asymptotic significance 1.9E-14). There was no association between the methylation index and the number of lymph nodes analyzed per patient (KruskalWallis). However, a statistically significant association between a positive lymph node biomarker panel status and the lymph node stage was observed. Of the 139 pN0 patients, only $10(7 \%)$ were biomarker panel positive, whereas 20 out of the $47(43 \%) \mathrm{pN} 1$ patients and as much as 14 out of the $15(93 \%) \mathrm{pN} 2$ patients were biomarker panel positive ( $P=8.2 \mathrm{E}-17$, Pearson's chisquare). A significant association was seen between the biomarker panel status in lymph nodes from cancer patients and the TNM stage, whereas $2 / 38$ (5\%) and 8/101 (8\%) of the patients with local disease (stages I and II,

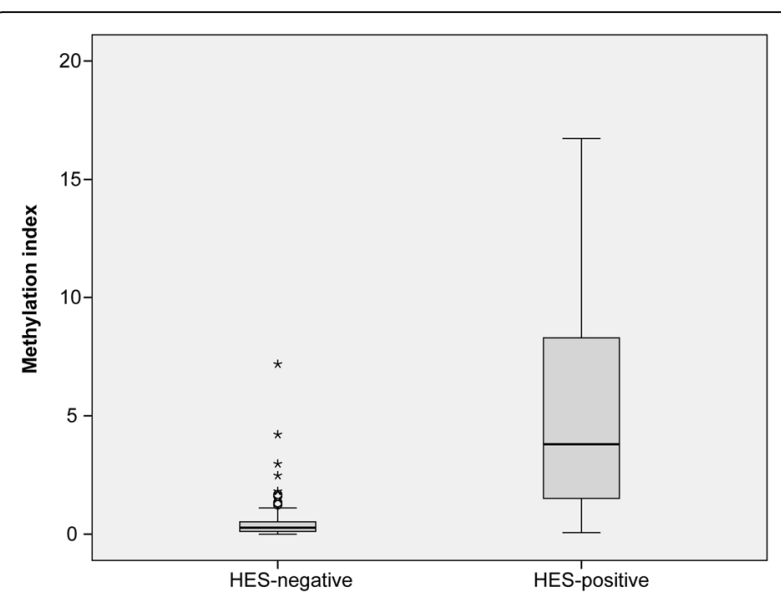

Fig. 3 Methylation index across the lymph nodes from colon cancer patients. HES-positives include patients for whom one or more of the lymph nodes subjected to methylation analysis were HES-positive. Abbreviation: HES, hematoxylin-erythrosin-safranin

respectively) were biomarker positive and as much as $34 / 62(55 \%)$ of the patients with regional disease (stage III) were positive ( $P=4.1 \mathrm{E}-13$; Pearson's chi-square).

\section{Prognostic relevance of the methylation biomarker panel} Within a median 5.0-year follow-up ( $\max 9.8$ years), there were 58 (29\%) deaths, including 26 (13\%) diseasespecific deaths among the 201 patients included in the study. Thirty-two (16\%) experienced relapse (distant metastases and/or locoregional relapses). Distant metastases were found in 29 (91\%) of these.

Among patients that were positive for the DNA methylation biomarker panel in the analyzed lymph nodes (22\%; 44 out of 201), 15/44 (34\%) experienced relapse or died from colorectal cancer. In contrast, only $17 / 157$ (11\%) of patients that were negative for the DNA methylation biomarker panel experienced relapse or died from the disease ( $P=1.9 \mathrm{E}-4$; Pearson's chi-square). This comparison included all patients and also stage III patients with HES-positive lymph nodes. Among the 139 lymph node-negative patients, $11(8 \%)$ experienced disease relapse. These patients were also negative for the DNA methylation biomarker panel. In addition to this dichotomous comparison, the methylation index as a continuous variable, as well as the methylation level of individual genes in the biomarker panel, was compared between pNO patients with and without relapse. No associations were found (Mann-Whitney $U$ ), underscoring that the detected methylation of sentinel lymph nodes was not suitable to differentiate between pN0 patients with and without relapse.

Based on the association between the biomarker panel status in lymph nodes from cancer patients and the TNM stage, it was not surprising that patients with methylation-positive lymph nodes had an inferior 5-year 
overall survival (50\% events) compared with patients with methylation-negative lymph nodes (23\% events; $P=3.0 \mathrm{E}-4$, Wald test; Fig. 4a) in univariate analysis. The lymph node methylation-positive patients also experienced more frequent recurrence (34\%) after 5 years follow-up than did those with negative lymph nodes (11\%; $P=3.1 \mathrm{E}-4$, Wald test; Fig. 4b).

When stratified according to stage, the same trends of inferior overall 5-year survival and more recurrences could be seen for stage III patients with methylationpositive lymph nodes, although this was not statistically significant $(P=1.4 \mathrm{E}-1$ and $P=6.7 \mathrm{E}-2$, respectively; Wald test; Fig. 4c, d). Survival analyses were not performed for early stage (I and II) patients, due to a limited number of methylation index-positive cases. Of importance, the distribution of the number of sentinel lymph nodes analyzed was equal among recurring and non-recurring patients, also when stratified according to stage (Mann-Whitney $U$ test, $P=9.6 \mathrm{E}-1$ ).
By using a stepwise selection procedure by Akaike information criterion (AIC), patient age and stage were identified as relevant predictor variables for stage III patients. From multivariate Cox regression, including these additional covariates, a trend similar to the univariate analysis could be seen for the prognostic value of the methylation index (overall survival, $P=1.3 \mathrm{E}-1$; time to recurrence, $P=8.4 \mathrm{E}-2$ ).

\section{Discussion}

Due to the high accuracy of the present DNA methylation biomarker panel for detecting malignant as well as benign colorectal tumors [13, 14], we aimed at investigating whether the panel could improve current lymph node diagnostics by detecting methylation in HESnegative lymph nodes from patients that later experienced relapse. To evaluate this, we analyzed the primary tumor in addition to a median of four sentinel lymph nodes from $\sim 200$ colon cancer patients, amounting to
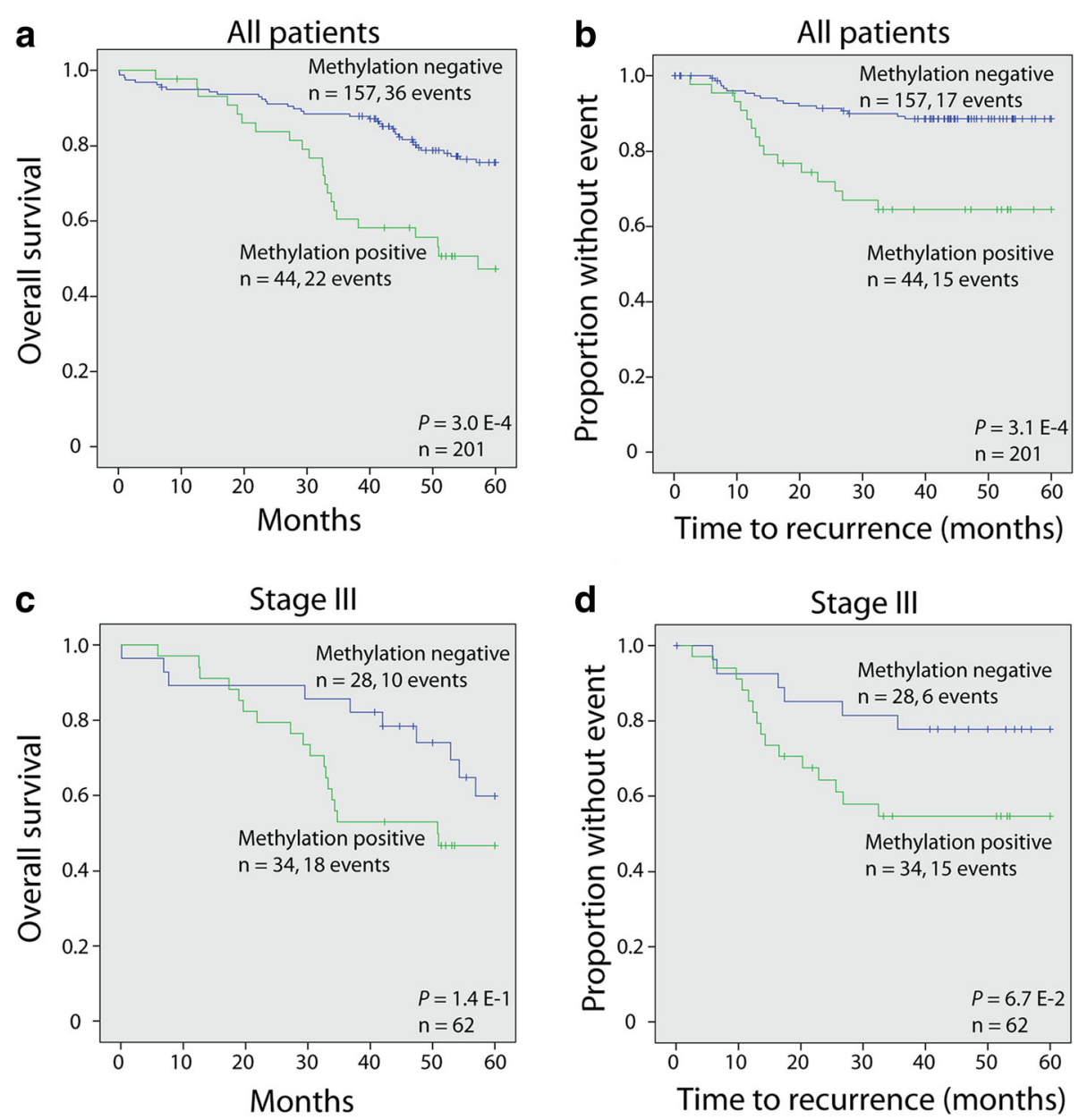

Fig. 4 Methylated lymph nodes are associated with poor patient outcome. a-d Survival analyses for methylation status of colon cancer patients' lymph nodes. Blue curves represent an unmethylated lymph node status whereas green curves represent a methylated lymph node status in colon cancer patients. a, c Overall survival analysis. b, d Time to recurrence analysis. The plots have been generated using the Kaplan-Meyer method, and $P$ values were calculated by the Wald test 
more than 1000 clinical samples. Indeed, compared to the number of lymph nodes that were histologically positive, a significantly higher number was methylationpositive, opening up the possibility that the methylation biomarkers could be more sensitive than HES staining alone in detecting small metastases. However, all $11 \mathrm{pN} 0$ relapsing patients in the dataset were also lymph node methylation-negative, and we were not able to identify the colon cancer patients that experienced relapse.

The aim of the sentinel node approach is to enable a more thorough analysis of those lymph nodes that are expected to have the highest risk of metastatic disease [7]. However, an extensive meta-analysis has demonstrated that the sentinel lymph node procedure has a low sensitivity for lymph node metastasis detection [15]. In a previous report analyzing the same clinical material used in the present study, detection of occult sentinel lymph node metastases by Cytokeratin 20 and Mucin 2 mRNA quantification had no prognostic value [16]. It cannot be ruled out that the low sensitivity of the sentinel lymph node procedure might have impacted the results of both studies. Interestingly, molecular tumor cell detection in regional lymph nodes, not restricted to sentinel lymph nodes, has been associated with poor overall and disease-free survival in meta-analyses [8]. Furthermore, in a recent prospective multicenter trial, a high molecularly determined micrometastasis volume in lymph nodes was shown to be an independent prognostic factor for poor overall and disease-free survival [17]. Thus, the lack of prognostic value for pN0 patients in the present study may be related to the fact that the DNA methylation analyses were restricted to the sentinel lymph nodes and not all available regional nodes.

The methylation level of patient's lymph nodes was evaluated using a methylation index, comprising the average PMR value across six genes and across all lymph nodes analyzed from a patient. This way, the methylation index was not affected by the number of lymph nodes analyzed per patient (ranging from 1 to 10). To exclude that a single methylation-positive lymph node was potentially "masked" in the methylation index by the presence of several methylation-negative lymph nodes, all analyzed lymph nodes among all pNO patients that experienced relapse were analyzed individually and confirmed to be negative. Although we could not detect occult metastases in the relapsing pN0 patients, survival analyses revealed a prognostic effect of the DNA methylation biomarkers in the overall cohort, which was highly significant in univariate analyses. The results were not statistically significant in multivariate analyses, but the clear trend indicated that significance could be achievable in a larger patient series.
Thirteen of the patients had methylation-positive sentinel lymph nodes that were negative by HES staining. Interestingly, at the patient level, three (23\%) of these patients had a positive lymph node metastasis status ( $\mathrm{pN} 1$ or $\mathrm{pN} 2$ ) - based on evaluation of other lymph nodes than those included for molecular analyses in the present study. This indicated that the methylation-positive lymph nodes might harbor metastases overlooked during the pathological evaluation or that the metastases were restricted to the half of the nodes that were subjected to methylation analysis. In such a context, the biomarker panel could be of added value in lymph node diagnostics.

For the analyses, we have used real-time quantitative methylation-specific PCR (qMSP; also called MethyLight) [18]. This is a streamlined and common method for methylation analyses, which can be easily standardized for large sample series [19]. Although the method is estimated to be able to detect 1 methylated allele among 10,000 unmethylated [18], it may not be sensitive enough for detecting potentially rare methylation events in lymph nodes that are negative in histological analyses. In these reactions, the rare methylated templates might be outcompeted by the highly abundant unmethylated DNA, possibly providing false negative results. An interesting alternative could be droplet digital PCR, where the amplification step is partitioned into thousands of droplets with individual PCRs. Using such sample dilution, the competition is significantly reduced, increasing the chances of detecting rare alleles [20]. Indeed, detection limits down to $0.001 \%$ have been reported [21]. Digital PCR is commonly used for copy number variation analysis and mutation/rare variant detection [22] but has recently been used as an alternative to realtime quantitative methylation-specific PCR [23-25].

\section{Conclusions}

In summary, we have demonstrated that the epigenetic biomarker panel analyzed here by real-time methylation-specific PCR showed a strong association with both HES staining and lymph node stage. Colon cancer patients with high sentinel lymph node methylation had an inferior prognosis, although not significant in multivariate analyses. Occult metastases in TNM stage II patients that experienced relapse were not detected.

\section{Methods}

\section{Clinical samples}

The present study comprised 1023 DNA samples from 201 colon cancer patients and 21 controls, consecutively collected at Stavanger University Hospital in the period of 2003 until 2010 [16, 26]. Clinical and genetic characteristics of the patients are listed in Table 1. 
Table 1 Clinical and genetic characteristics of colon cancer patients and tumors

\begin{tabular}{|c|c|c|}
\hline & $\begin{array}{l}\text { Number of patients } \\
(n=201)\end{array}$ & $\begin{array}{l}\text { Percentage } \\
(\%)\end{array}$ \\
\hline \multicolumn{3}{|l|}{ Stage } \\
\hline I & 38 & 19 \\
\hline ॥ & 101 & 50 \\
\hline III & 62 & 31 \\
\hline \multicolumn{3}{|l|}{ N stage } \\
\hline 0 & 139 & 69 \\
\hline 1 & 47 & 23 \\
\hline 2 & 15 & 7 \\
\hline \multicolumn{3}{|l|}{ Grade } \\
\hline Low & 50 & 25 \\
\hline Moderate & 141 & 70 \\
\hline High & 10 & 5 \\
\hline \multicolumn{3}{|l|}{ Localization } \\
\hline Proximal & 129 & 64 \\
\hline Distal & 72 & 36 \\
\hline \multicolumn{3}{|l|}{ Gender } \\
\hline Female & 112 & 56 \\
\hline Male & 89 & 44 \\
\hline \multicolumn{3}{|l|}{ Age } \\
\hline$<70$ & 60 & 30 \\
\hline \multirow[t]{2}{*}{$\geq 70$} & 141 & 70 \\
\hline & $\begin{array}{l}\text { Number of cancers } \\
(n=197)\end{array}$ & $\begin{array}{l}\text { Percentage } \\
(\%)\end{array}$ \\
\hline
\end{tabular}

MSI status

\begin{tabular}{ccc} 
MSI & 61 & 31 \\
MSS & 136 & 69 \\
BRAF status & & 27 \\
Mutation & 52 & 73 \\
Wild type & 144 & \\
\hline
\end{tabular}

The MSS group includes both MSS and MSI-low samples. BRAF status is evaluated for the V600E hotspot. For one cancer, BRAF status could not be determined MSI microsattelite instability, MSS microsattelite stable

\section{Sentinel lymph node sampling}

Ex vivo sentinel lymph node mapping was performed as previously described [26]. The lymph nodes were divided in two. One part was formalin fixed and subjected to staining with hematoxylin-erythrosin-safranin (HES) for routine histological evaluation [27]. The second part was snap-frozen in liquid nitrogen and stored at $-80{ }^{\circ} \mathrm{C}$ for molecular analyses [26].

\section{Test and validation series}

From the colon cancer patients, 197 primary tumors and 783 sentinel lymph nodes were available for molecular analyses (median 4 lymph nodes per patient; range 1 to 10). Control samples included 43 normal lymph nodes from 21 patients (median 2 lymph nodes per patient; range 1 to 4 ) with benign disease undergoing bowel resection. The clinical material was divided into a test and validation series. The test series was randomly selected from the consecutive series and comprised 77 colon cancer patients, including 75 primary carcinomas, 292 patient lymph nodes, and 26 normal lymph node controls. The remaining validation series counted 124 patients and included 122 primary carcinomas, 491 patient lymph nodes, and 17 normal lymph node controls. The test series was used to set thresholds for methylation scoring. In subsequent statistical analyses, the two series were pooled for robustness.

\section{DNA isolation and promoter methylation analyses}

DNA from tumor samples and lymph nodes was extracted using the DNeasy Mini Kit (Qiagen; first 129 patients) or the Allprep RNA/DNA kit (Qiagen), as previously described [16]. For each sample, $1.3 \mu \mathrm{g}$ DNA was bisulfite treated using the EpiTec Bisulfite kit (Qiagen). DNA desulfonation and purification was performed automatically, using a QiaCube (Qiagen), and the DNA was eluted in $40 \mu \mathrm{l}$ elution buffer. The promoter methylation status of the six genes CNRIP1, FBN1, INA, MAL, $S N C A$, and SPG20 comprising the biomarker panel was analyzed individually by quantitative methylationspecific PCR (qMSP) as previously described [13, 14]. Briefly, samples were analyzed in triplicates in 384 well plates generated by a pipetting robot (EpMotion 5075, Eppendorf). The reaction volume in each well was $20 \mu \mathrm{l}$ comprising $30 \mathrm{ng}$ bisulfite-treated DNA, $0.9 \mu \mathrm{M}$ each of the forward and the reverse primer, $0.2 \mu \mathrm{M}$ 6-FAM labeled probe [14] with a minor groove binder nonfluorescent quencher, and $1 \times$ TaqMan Universal PCR master mix NoAmpErase UNG (Life Technologies). A standard curve was generated using a 1:5 serial dilution (32.5-0.052 ng) of commercially in vitro methylated DNA (IVD; CpGenome Universal Methylated DNA; Millipore) and was used to determine the quantity from the resulting quantification cycle $(\mathrm{Cq})$ values. For all samples, $\mathrm{Cq}$ values above 35 were censored, according to the recommendations from the manufacturer (Life Technologies). The standard curve was included in each 384 well plate along with a methylated control (IVD), an unmethylated control (normal blood), and a negative control (water). For normalization of possible variation in bisulfite-treated DNA input, the ALU-C4 repeat was used [28]. All 384 well plates were amplified using the 7900HT Sequence Detection System (TaqMan; Life Technologies); $95^{\circ} \mathrm{C}$ for $10 \mathrm{~min}$, then 45 cycles of $95^{\circ} \mathrm{C}$ for $15 \mathrm{~s}$ followed by $60{ }^{\circ} \mathrm{C}$ for $1 \mathrm{~min}$. For each sample, the median quantity of the triplicates was used to 
calculate the percentage of methylated molecules per sample (PMR value) [19] by normalizing the markerspecific quantity to the ALU-C4 repeat value, relative to the same ratio for the fully methylated control (IVD).

\section{Definition of biomarker positivity}

Colon cancers were considered DNA methylation biomarker panel positive when a minimum of two of the six biomarkers analyzed had a PMR value $\geq 7$, as previously described [14]. For individual lymph nodes, the mean PMR value across the biomarker panel was calculated. At the patient level, the lymph node methylation status was determined by calculating a methylation index: the average PMR value across all genes in the biomarker panel and across all lymph nodes analyzed from the patient in question. To dichotomize the results, for individual lymph nodes and per patient, into methylated and unmethylated groups, scoring thresholds were determined from the test series (see the "Results" section).

\section{Statistical analyses}

Analyses were performed with the IBM SPSS statistics 21 software (IBM, New York, USA) and R 3.2.2 using the "survival" package [29]. Pearson's chi-square and Fisher's exact tests were run to evaluate independence between categorical variables. ROC curves were used to assess the accuracy of the methylation status to differentiate between (1) lymph nodes with negative or positive HES status and (2) patients with negative or positive HES lymph node staining, restricted to the lymph nodes analyzed for methylation. The accuracy is expressed as the area under the ROC curve (AUC). The state variable was HES positivity. Potential differences in the methylation index were further evaluated by the Kruskal-Wallis and Mann-Whitney $U$ tests. $P$ values were derived from two-sided tests using a significance level of 0.05 .

For survival analyses, the Kaplan-Meier method was used to generate survival plots. In agreement with previously presented guidelines [30], overall survival was defined as the time from surgery to death of any cause. Time to recurrence was accordingly defined as the time from surgery to the first event of either death from the same cancer, locoregional recurrence, or distant metastases [30]. The survival curves were generated using dichotomized methylation values and $P$ values derived from a Wald test. To evaluate whether lymph node methylation had significant independent impact on patient survival, multivariate Cox regression was used. Relevant predictor variables for the Cox regression model were identified by using a stepwise selection procedure by Akaike information criterion (AIC) [31].

\section{Additional file}

Additional file 1: Figure S1. Venn diagram illustrating the overlap between HES-positive and DNA methylation biomarker-positive lymph nodes. (A) Individual lymph node level. (B) Patient level. Abbreviation: HES, hematoxylin-erythrosin-safranin. (PDF 146 kb)

\section{Abbreviations}

AUC: Area under the receiver operating characteristics curve; Cl: Confidence interval; HES: Hematoxylin-erythrosin-safranin; IVD: In vitro methylated DNA; PMR: Percentage methylated reference (methylation value); ROC: Receiver operating characteristics

\section{Acknowledgements}

Not applicable

\section{Funding}

This study was funded by the Western Norway Health Authorities, Stiftelsen Kristian Gerhard Jebsen, the Norwegian Cancer Consortium, and the Folke Hermansen Fund. The work was partly supported by the Research Council of Norway through its Centre of Excellence funding scheme, project number 179571.

Availability of data and materials

The datasets used and/or analyzed during the current study are available from the corresponding author on reasonable request.

\section{Authors' contributions}

RAL and ON were responsible for the conception and design. GEL, MG, TA, $I H, K S, H K$, and ON were responsible for the acquisition of data. GEL, MG, TA, $\mathrm{MJ}, \mathrm{RAL}$, and $\mathrm{ON}$ were responsible for the analyses and interpretation of data. GEL was responsible for the drafting of the manuscript. All authors read and approved the final manuscript.

Ethics approval and consent to participate

Informed written consent was obtained from all patients, and the study was approved by the Regional Committees for Medical and Health Research Ethics (REC 197.04). All methods were performed in accordance with the relevant guidelines and regulations.

\section{Consent for publication}

Not applicable

\section{Competing interests}

The authors declare that they have no competing interests.

\section{Publisher's Note}

Springer Nature remains neutral with regard to jurisdictional claims in published maps and institutional affiliations.

\footnotetext{
Author details

${ }^{1}$ Department of Molecular Oncology, Institute for Cancer Research, The Norwegian Radium Hospital, Oslo University Hospital, PO Box 4950 Nydalen, 0424 Oslo, Norway. ${ }^{2}$ Centre for Cancer Biomedicine, Faculty of Medicine, University of Oslo, Oslo, Norway. ${ }^{3}$ K.G Jebsen Colorectal Cancer Research Centre, Oslo University Hospital, Oslo, Norway. ${ }^{4}$ Department of Chemistry, Biotechnology, and Food science, Norwegian University of Life Sciences, Ås, Norway. ${ }^{5}$ Department of Hematology and Oncology, Stavanger University Hospital, PO Box 8100, 4068 Stavanger, Norway. ${ }^{6}$ Department of Gastrointestinal Surgery, Stavanger University Hospital, Stavanger, Norway. ${ }^{7}$ Department of Clinical Medicine, University of Bergen, Bergen, Norway. ${ }^{8}$ Gastrointestinal Translational Research Unit, Laboratory for Molecular Medicine, Stavanger University Hospital, Stavanger, Norway.
} 
Received: 6 May 2017 Accepted: 28 August 2017

\section{Published online: 05 September 2017}

\section{References}

1. Ferlay J, Soerjomataram I, Dikshit R, Eser S, Mathers C, Rebolo M, et al. Cancer incidence and mortality worldwide: sources, methods and major patterns in GLOBOCAN 2012. Int J Cancer. 2015;136:E359-86.

2. Dienstmann $R$, Salazar $R$, Tabernero J. Personalizing colon cancer adjuvant therapy: selecting optimal treatments for individual patients. J Clin Oncol. 2015;33:1787-96.

3. Sinicrope FA, Okamoto K, Kasi PM, Kawakami H. Molecular biomarkers in the personalized treatment of colorectal cancer. Clin Gastroenterol Hepatol. 2016;14:651-8.

4. Veen T, Nedrebø BS, Stormark K, Søreide JA, Kørner H, Søreide K. Qualitative and quantitative issues of lymph nodes as prognostic factor in colon cancer. Dig Surg. 2013;30:1-11.

5. Benson ABIII, Schrag D, Somerfield MR, Cohen AM, Figueredo AT, Flynn PJ, et al. American Society of Clinical Oncology recommendations on adjuvant chemotherapy for stage II colon cancer. J Clin Oncol. 2004;22:3408-19.

6. Gray R, Barnwell J, McConkey C, Hills RK, Williams NS, Kerr DJ. Adjuvant chemotherapy versus observation in patients with colorectal cancer: a randomised study. Lancet. 2007;370:2020-9.

7. Weitz J, Koch M, Debus J, Höhler T, Galle PR, Büchler MW. Colorectal cancer. Lancet. 2005;365:153-65.

8. Rahbari NN, Bork U, Motschall E, Thorlund K, Büchler MW, Koch M, Weitz J. Molecular detection of tumor cells in regional lymph nodes is associated with disease recurrence and poor survival in node-negative colorectal cancer: a systematic review and meta-analysis. J Clin Oncol. 2012;30:60-70.

9. Okugawa Y, Grady WM, Goel A. Epigenetic alterations in colorectal cancer: emerging biomarkers. Gastroenterology. 2015;149:1204-25.

10. Ahlquist T, Lind GE, Costa VL, Meling Gl, Vatn M, Hoff GS, et al. Gene methylation profiles of normal mucosa, and benign and malignant colorectal tumors identify early onset markers. Mol Cancer. 2008;7:94.

11. Ahmed D, Danielsen SA, Aagesen TH, Bretthauer M, Thiis-Evensen E, Hoff G, et al. A tissue-based comparative effectiveness analysis of biomarkers for early detection of colorectal tumors. Clin Transl Gastroenterol. 2012;e27:3.

12. Lind GE, Ahlquist T, Lothe RA. DNA hypermethylation of MAL: a promising diagnostic biomarker for colorectal tumors. Gastroenterology. 2007;132:1631-2.

13. Lind GE, Raiborg C, Danielsen SA, Rognum TO, Thiis-Evensen E, Hoff G, et al $S P G 20$, a novel biomarker in colorectal carcinogenesis, encodes a regulator of cytokinesis. Oncogene. 2011;30:3967-78.

14. Lind GE, Danielsen SA, Ahlquist T, Merok M, Andresen K, Skotheim Rl, et al. Identification of an epigenetic biomarker panel with high sensitivity and specificity for colorectal cancer and adenomas. Mol Cancer. 2011;10:85.

15. van der Pas MH, Meijer S, Hoekstra OS, Riphagen II, de Vet HC, Knol DL, et al. Sentinel-lymph-node procedure in colon and rectal cancer: a systematic review and meta-analysis. Lancet Oncol. 2011;12:540-50.

16. Nordgard O, Oltedal S, Aasprong OG, Søreide JA, Søreide K, Tjensvoll K, et al. Prognostic relevance of occult metastases detected by cytokeratin 20 and mucin 2 mRNA levels in sentinel lymph nodes from colon cancer patients. Ann Surg Oncol. 2012;19:3719-26.

17. Yamamoto H, Murata K, Fukunaga M, Ohnishi T, Noura S, Miyake Y, et al. Micrometastasis volume in lymph nodes determines disease recurrence rate of stage II colorectal cancer: a prospective multicenter trial. Clin Cancer Res. 2016;22:3201-8

18. Eads CA, Danenberg KD, Kawakami K, Salz LB, Blake C, Shibata D, et al. MethyLight: a high-throughput assay to measure DNA methylation. Nucleic Acids Res. 2000;28:E32.

19. Pharo HD, Honne H, Vedeld HM, Dahl C, Andresen K, Liestøl K, et al. Experimental factors affecting the robustness of DNA methylation analysis. Sci Rep. 2016;6:33936.

20. Vogelstein B, Kinzler KW. Digital PCR. Proc Natl Acad Sci U S A. 1999;96:9236-41.

21. Hindson BJ, Ness KD, Masquelier DA, Belgrader P, Heredia NJ, Makerewicz AJ, et al. High-throughput droplet digital PCR system for absolute quantitation of DNA copy number. Anal Chem. 2011;83:8604-10.

22. Day E, Dear PH, McCaughan F. Digital PCR strategies in the development and analysis of molecular biomarkers for personalized medicine. Methods. 2013;59:101-7

23. Weisenberger DJ, Trinh BN, Campan M, Sharma S, Long TI, Ananthnarayan $S$, et al. DNA methylation analysis by digital bisulfite genomic sequencing and digital MethyLight. Nucleic Acids Res. 2008;36:4689-98.
24. Yu M, Carter KT, Makar KW, Vickers K, Ulrich CM, Schoen RE, et al. MethyLight droplet digital PCR for detection and absolute quantification of infrequently methylated alleles. Epigenetics. 2015;10:803-9.

25. Wiencke JK, Butler R, Hsuang G, Eliot M, Kim S, Sepulveda MA, et al. The DNA methylation profile of activated human natural killer cells. Epigenetics. 2016;11:363-80.

26. Nordgard O, Oltedal S, Kørner H, Aasprong OG, Tjensvoll K, Gilje B, Heikkila R. Quantitative RT-PCR detection of tumor cells in sentinel lymph nodes isolated from colon cancer patients with an ex vivo approach. Ann Surg 2009;249:602-7.

27. Edston E, Gröntoft L. Saffron-a connective tissue counterstain in routine pathology. J Histotechnol. 1977;20:123-5.

28. Weisenberger DJ, Campan M, Long TI, Kim M, Woods C, Fiala E. Analysis of repetitive element DNA methylation by MethyLight. Nucleic Acids Res. 2005;33:6823-36.

29. Therneau TM, Grambsch PM. Modeling survival data: extending the cox model: Springer; 2000. http://www.springer.com/us/book/9780387987842.

30. Punt CJ, Buyse M, Kohne CH, Hohenberger P, Labianca R, Schmoll HJ, et al. Endpoints in adjuvant treatment trials: a systematic review of the literature in colon cancer and proposed definitions for future trials. J Natl Cancer Inst. 2007;99:998-1003.

31. Rizopoulos D. Bootstrap StepAIC in model selection by bootstrapping the stepAIC() procedure. 2009 .

\section{Submit your next manuscript to BioMed Central and we will help you at every step:}

- We accept pre-submission inquiries

- Our selector tool helps you to find the most relevant journal

- We provide round the clock customer support

- Convenient online submission

- Thorough peer review

- Inclusion in PubMed and all major indexing services

- Maximum visibility for your research

Submit your manuscript at www.biomedcentral.com/submit
C Biomed Central 